\title{
Microarray analysis of NSAIDs-treated cardiomyocytes to search for genes involved in COX-2 inhibitor cardiotoxicity
}

\author{
C.K. Oh ${ }^{1,2}$, M.S.F. Ahmed ${ }^{1}$, C. Xie ${ }^{1}$, J.H. Ryu ${ }^{1}$, J. Choi ${ }^{3}$, J.S. Chung ${ }^{2}$, \\ G.S. Roh ${ }^{1}$, H.J. Kim ${ }^{1}$, G.J. Cho ${ }^{1}$, W.S. Choi ${ }^{1}$, S.S. Kang ${ }^{1}$, D.H. Lee ${ }^{1}$ \\ ${ }^{1}$ Department of Anatomy \& Convergence Medical Science, Institute of \\ Health Sciences, College of Medicine, Gyeongsang National University, \\ Jinju, Korea \\ ${ }^{2}$ Department of Urology, Haeundae Paik Hospital, Inje University College \\ of Medicine, Busan, Korea. \\ ${ }^{3}$ Gyeongnam Department of Environmental Toxicology and Chemistry, \\ Korea Institute of Toxicology, Jinju, Korea.
}

Corresponding author: D.H. Lee

E-mail: bx113@gnu.ac.kr

Genet. Mol. Res. 17 (4): gmr18068

Received June 26, 2018

Accepted November 12, 2018

Published December 13, 2018

DOI http://dx.doi.org/10.4238/gmr18068

\begin{abstract}
Non-steroidal anti-inflammatory drugs (NSAIDs) are widely-used medications for the treatment of many inflammatory diseases. . They inhibit cyclooxygenase (COX) enzymes as a primary mechanism of action. However, many of these drugs were withdrawn from the market because of cardiovascular side effects. To date, only a limited number of genes and specific gene variants that account for their cardiac toxicity have been investigated. To identify possible cardiotoxic effects, we performed a microarray analysis of NSAIDs-treated primary cardiomyocytes. Primary cultures of neonatal rat cardiomyocytes were established and a DNA microarray was run on extracted RNA from celecoxib and diclofenac-treated cardiomyocytes. The changes in gene expression for all potentially predisposing variants were investigated. In cells treated with celecoxib, the expression of 1718 and 560 genes decreased and increased respectively by 2 -fold or more. When cells were treated with diclofenac, the expression of 424 and 32 genes decreased and increased respectively by 2 -fold or more. NSAIDs affected the expression of genes involved in calcium and potassium signaling.
\end{abstract}


Pathway analysis of gene expression of NSAIDs-treated cardiomyocytes showed changes in gene expression involving major pathways, including apoptosis, signal transduction and transcription. These findings provide a clue to explain, at least in part, how NSAIDs provoke side effects in the heart. These data could be used to elect target genes for studying cardiotoxic effects of NSAIDs and for developing new drugs.

Key words: Cardiotoxicity; Celecoxib; Diclofenac; Microarray; NSAIDs; EC decoupling

\section{INTRODUCTION}

Non-steroidal anti-inflammatory drugs (NSAIDs) are widely used as antiinflammatory and analgesic drugs. NSAIDs inhibit cyclooxygenase (COX) enzymes as a primary mechanism of action (Palayoor et al., 2012). Recent evidence indicates that NSAIDs interfere with a variety of cellular processes, including signal transduction, transcription, DNA repair and cell-cycle progression (Jones et al., 1999; Lin et al., 2004; Raju et al., 2002; Tegeder et al., 2001; Tseng et al., 2002; Wei et al., 2004). NSAIDs have received considerable attention in cancer and pre-clinical studies because of their anti-tumor effects (Yin et al., 2016).

Nonspecific COX inhibitors can cause gastric and renal toxicity, attributed to the inhibition of COX-1- derived prostaglandins (Wolfe et al., 1999). Selective COX-2 inhibitors, such as diclofenac and celecoxib, were introduced later and had less gastrointestinal side effects but they have been associated with a higher risk of cardiovascular events compared to the dual specific COX inhibitors (Brophy, 2005; Ferraz et al., 2000; Wolfe et al., 1999). Even short-term usage of NSAIDs increases the risk of recurrence of myocardial infarction (Schjerning Olsen et al., 2011). To date, few studies have investigated the cardiotoxic effects of selective COX-2 NSAIDs and even fewer have provided a comprehensive evaluation of a probable genetic basis.

We treated primary cultures of neonatal rat cardiomyocytes with celecoxib or diclofenac and then analyzed their global gene expression using a microarray assay versus a no treatment control. Our objective was to provide a comprehensive list of potential off-target genes involved in the cardiotoxicity of NSAIDs. We focused on changes in physiological pathways, calcium $\left(\mathrm{Ca}^{+2}\right)$ and potassium $\left(\mathrm{K}^{+}\right)$channels.

\section{MATERIAL AND METHODS}

\section{Animals and materials}

Sprague Dawley rats were purchased from the Koatech Central Laboratory (Pyeongtaek, Korea). Dulbecco's minimal essential medium (DMEM) was purchased from Gibco (Carlsbad, CA, USA). Reagents used in the preparation and analysis of the microarray were purchased from Agilent Technologies (Santa Clara, CA). All other reagents and antibodies were purchased from Sigma-Aldrich (St. Louis, MO). 


\section{Cardiomyocyte primary culture preparation}

One-day old rats were anesthetized using ether, and ventricles were harvested and collected in 1X PBS. A 1-3 mm minced portion of each sample was briefly centrifuged to remove the remaining blood cells, shaken on ice for 20 minutes and then transferred to a 40 $\mathrm{ml}$ conical flask filled with $0.08 \%$ trypsin solution. The flask was centrifuged at $2000 \mathrm{rpm}$ at $37^{\circ} \mathrm{C}$ for 10 minutes and briefly precipitated on ice. The supernatant was transferred to a $15 \mathrm{ml}$ tube and centrifuged at $10000 \mathrm{rpm}$ for 5 minutes. Trypsin digestion was repeated 2-3 times depending on the amount of tissue remains in the supernatant. Primary cultures were established on DMEM medium containing 10\% fetal bovine serum (FBS), 100 units $/ \mathrm{ml}$ penicillin and $100 \mathrm{mg} / \mathrm{ml}$ streptomycin and then incubated at $37^{\circ} \mathrm{C} / 5 \% \mathrm{CO}_{2}$ for $24 \mathrm{~h}$ as described in Rodgers et al. (2009). Animals were maintained according to the guideline for the care and use of laboratory animals. Experimental protocol was approved by the Institutional Animal Care and Use Committee of Gyeongsang National University (Jinju, Republic of Korea).

\section{NSAIDs treatment}

IC50s were obtained for celecoxib and diclofenac using hERG, MTT assays, and electrophysiology and final concentrations were determined (16 uM) for celecoxib and (40 uM) for diclofenac (Baek et al., 2010; Yarishkin et al., 2009). Cultures were treated with either drug using $1,1 / 5$ or $1 / 25$ of the reference dose and for $6 \mathrm{~h}$ or $24 \mathrm{~h}$; six samples were run for each drug (three doses and two time points). The other experiments were run in triplicate; two controls of untreated cardiomyocytes were used for each drug.

\section{Microarray analysis}

Total RNA was isolated using TRIZOL reagent. A portion of the RNA was electrophoresed on an -agarose-formaldehyde gel to verify RNA quality according to the reference value used for the cDNA microarray with a NanoDrop spectrophotometer (NanoDrop Technologies, Delaware, USA). Samples with RNA ratio > 1.8 (OD 260/280) were considered adequate for the microarray analysis. The Agilent Total RNA Nano chip assay (Agilent Technologies, Palo Alto, CA) was used along with (Agilent Bioanalyzer 2100 ) to run the array. Images were acquired with GenePix Pro 6.0 software (Axon Instruments, Union City, CA).

\section{Image and statistical analysis}

The one-color images were scanned using Agilent's DNA microarray scanner and quantified with Feature Extraction Software (Agilent Technologies, USA). Extracted data were processed using GeneSpring GX 7.3 software (Axon Instruments, Union City, CA) to obtain normalized ratios (fold changes), clustering of gene expression and pathway analysis. Loess regression was used as described in Smyth and Speed, (2003) to adjust against systematic errors. Relative gene expression of celecoxib and diclofenac-treated cardiomyocytes compared to untreated controls ( $>2$ fold change) were considered significant using Welch's t-test when $\mathrm{p}<0.05$ ). 


\section{RESULTS}

\section{Changes in gene expression of celecoxib and diclofenac-treated cardiomyocytes}

We analyzed genes expression of neonatal rat cardiomyocytes using a DNA microarray assay. Out of 40,012 genes, from cells treated with celecoxib, the expression of 1718 and 560 genes decreased and increased respectively by 2 -fold or more. When cells were treated with diclofenac, the expression of 424 and 32 genes decreased and increased respectively by 2 -fold or more. Figure 1 shows a hierarchical cluster dendrogram of gene expression changes in celecoxib- and diclofenac-treated cardiomyocytes, and a complete dataset of normalized and raw microarray data can be found at NCBI's Gene Expression Omnibus (Barrett and Edgar, 2006) * and is accessible through GEO Series accession number GSE77591 for celecoxib (https://www.ncbi.nlm.nih.gov/geo/query/acc.cgi?acc=GSE77591) and GSE77880 for diclofenac (https://www.ncbi.nlm.nih.gov/geo/query/acc.cgi?acc=GSE77880).

A

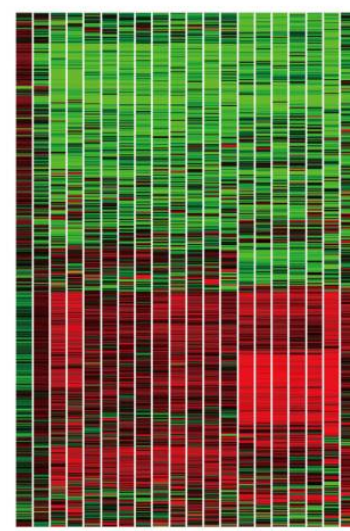

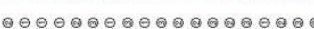

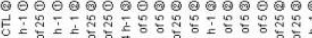

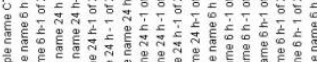

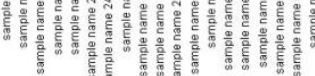

C

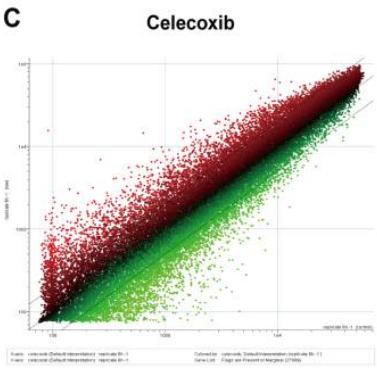

B

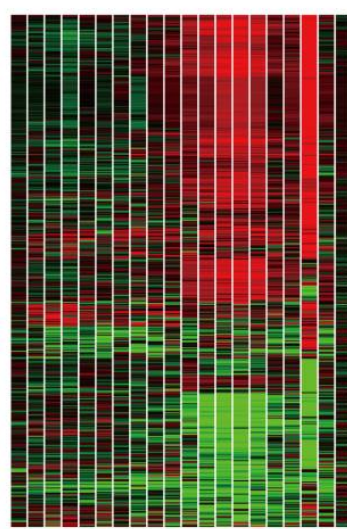

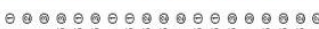

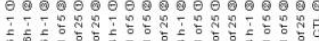

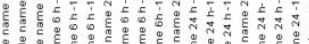

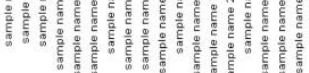

D

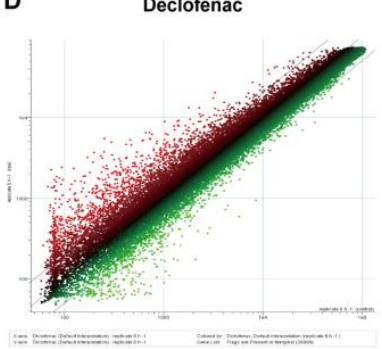

Figure 1. Hierarchical clustering of genes expression of celecoxib-treated (A) and diclofenac-treated (B) rat cardiomyocytes. Rat cardiomyocytes were treated with three different doses $(1,1 / 5$ or $1 / 25$ of the reference dose) for $6 \mathrm{~h}$ or $24 \mathrm{~h}$. Columns represent values obtained from the different experiments (two controls and triplicates of six different samples) and rows show differential gene expression as color variation (red: up-regulated expression; green: down-regulated expression). Scatter plot of DNA microarrays from the Celecoxib-treated (C) and Diclofenac-treated (D) rat primary cardiomyocytes. (6h, 1 of the reference dose). 
Treating cardiomyocytes using different doses and for different durations induced variable gene expression patterns (data not shown). In general, the smaller the dose the fewer the altered genes, which were also included in the high dose effect. However, we observed a wider effect of small doses when cells were treated for longer (Fig. 1 A, B). Celecoxib produced a broader scatter plot than Diclofenac (Fig. 1 C, D).

\section{Pathway analysis}

Pathway analysis of gene expression of celecoxib and diclofenac-treated cardiomyocytes showed changes in major pathways, including: apoptosis, interleukin binging, signal transduction, immune response and transcription. The numbers of altered gene expression patterns were comparable between the two drugs though the patterns differed, suggesting different mechanisms of action.

Genes encoding receptors and intermediators of MAPK (mitogen-activated protein kinase), among other pathways, were altered in celecoxib- and diclofenac-treated cardiomyocytes. These changes in gene expression included up-regulation of TGFBR (transforming growth factor beta receptor), Ras and NF-kB (nuclear factor kappa-light-chainenhancer of activated B cells) and down-regulation of FGFR (fibroblast growth factor receptor), PKA (protein kinase A) and PTP (protein tyrosine phosphatase). Tegeder et al. (2001) reported that NSAIDs interfere with transcription factors through similar intermediates.

\section{Effects on calcium signaling}

Treating cardiomyocytes with celecoxib and diclofenac generated changes in the expression of genes involved in $\mathrm{Ca}^{+2}$ signaling. Celecoxib (Table 1) and diclofenac (Table 2) induced off-target changes of different genes, suggesting different mechanisms. Celecoxib induced a wider effect on gene expression in terms of number and diversity, including genes expressing voltage dependent $\mathrm{Ca}^{+2}$ channels, $\mathrm{N}$ type, protein kinases and neurotransmitters receptors. However, most changes occurred in genes encoding receptors of regulatory cues in the cardiovascular system, including vasopressin, angiotensin II, cholinergic and prostaglandin E in the case of celecoxib, and only coagulation factor II and neurotensin in the case of diclofenac.

Table 1. List of greatly altered ( $>3$-fold change) or little altered $(<0.33$-fold change) expression of genes related to calcium channels in celecoxib-treated rat cardiomyocytes

\begin{tabular}{lccc}
\hline Gene symbol & Gene bank Acc. No & Fold change & Gene name \\
\hline Ptger1 & NM_013100 & 0.09 & prostaglandin E receptor 1 \\
5Ht-2 & AF203812 & 0.18 & 5-hydroxytryptamine (serotonin) receptor 2A \\
Gna15 & NM_053542 & 0.21 & guanine nucleotide binding protein, alpha 15 \\
IP3R3X & NM_013138 & 0.22 & inositol 1,4,5-triphosphate receptor 3 \\
Avpr1b & NM_017205 & 0.23 & arginine vasopressin receptor 1B \\
Gprc1a & Y18810 & 0.25 & glutamate receptor, metabotropic 1 \\
Ck2b & NM_021739 & 0.27 & calcium/calmodulin-dependent protein kinase II, beta \\
Erbb2 & M61004 & 0.28 & v-erb-b2 erythroblastic leukemia viral oncogene homolog 2 \\
bNOS & NM_052799 & 0.30 & nitric oxide synthase 1, neuronal \\
Agtr1a & NM_030985 & 0.31 & angiotensin II receptor, type 1 (AT1A) \\
Chrm1 & AJ006522 & 0.31 & cholinergic receptor, muscarinic 1 \\
Cacnalb & NM_147141 & 3.21 & protein kinase C, beta 1 \\
Pkcb & NM_012713 & 4.35 & calcium channel, voltage-dependent, N type, alpha 1B subunit \\
\hline
\end{tabular}


Table 2. List of altered ( $>3$-fold change or $<0.33$-fold change) expression of genes related to calcium channels in diclofenac-treated rat cardiomyocytes

\begin{tabular}{lccc}
\hline Gene symbol & Gene bank Acc. No. & Fold change & Gene name \\
\hline TRGPC & NM_012950 & 0.30 & coagulation factor II (thrombin) receptor \\
Ntsr1 & XM_345484 & 3.21 & neurotensin receptor 1 (predicted) \\
\hline
\end{tabular}

\section{Effects on potassium signaling}

Both drugs induced changes in the expression of genes involving $\mathrm{K}^{+}$channel families and subfamilies such as $\mathrm{V}$, shal-related, shaker-related and $\mathrm{K}$ subfamilies in the case of celecoxib; shaw-related and 12 subfamilies in the case of diclofenac (Tables 3 and 4). Both induced changes in members of the $\mathrm{H}$ and $\mathrm{M}$ subfamilies. Celecoxib impacted the $\mathrm{KCN}\left(\mathrm{K}^{+}\right.$ channels) family, which is related to regulatory functions in the neural and cardiovascular systems (Mackie and Byron, 2008).

Table 3. List of altered ( $>3$-fold change or $<0.33$-fold change) genes related to potassium channels in celecoxibtreated rat cardiomyocytes

\begin{tabular}{lccc}
\hline Gene symbol & Gene bank Acc. No. & Fold change & Gene name \\
\hline Kcnv2 & XM_220024 & 0.10 & $\begin{array}{c}\text { potassium channel, subfamily V, member 2 } \\
\text { Kcnmb1 }\end{array}$ \\
Kcc2 & NM_019273 & 0.11 & potassium large conductance calcium-activated channel, subfamily M, beta member \\
Kcnip2 & NM_134363 & 0.17 & solute carrier family 12, (potassium-chloride transporter) member 5 \\
5Ht-2 & XM_574676 & 0.17 & Kv channel-interacting protein 2 \\
Nckx3 & AF203812 & 0.18 & 5-hydroxytryptamine (serotonin) receptor 2A \\
Fxyd4 & XM_342533 & 0.19 & solute carrier family 24 (sodium/potassium/calcium exchanger), member 3 \\
Kcnh6 & NM_022388 & 0.19 & FXYD domain-containing ion transport regulator 4 \\
Tresk-2 & NM_053937 & 0.25 & potassium voltage-gated channel, subfamily H (eag-related), member 6 \\
Kcnip1 & NM_001003820 & 0.26 & tandem-pore K+ (K(2P)) channel \\
Kcna & AY142709 & 0.28 & Kv channel-interacting protein 1 \\
Kcnd3 & NM_173095 & 0.29 & potassium voltage-gated channel, shaker-related subfamily, member 1 \\
Kchip1 & NM_031739 & 0.30 & potassium voltage gated channel, Shal-related family, member 3 \\
Kcnk13 & NM_022929 & 0.30 & Kv channel-interacting protein 1 \\
Kctd13 & NM_022293 & 3.21 & potassium channel, subfamily K, member 13 \\
Kcnk1 & NM_198736 & 4.8 & potassium channel tetramerization domain containing 13 \\
\hline
\end{tabular}

Table 4. List of altered ( $>3$-fold change or $<0.33$-fold change) expression of genes related to potassium channels in diclofenac-treated rat cardiomyocytes

\begin{tabular}{lccc}
\hline Gene symbol & $\begin{array}{c}\text { Gene bank } \\
\text { Acc. No. }\end{array}$ & Fold change & Gene name \\
\hline KShIIID & NM_053997 & 0.18 & $\begin{array}{c}\text { potassium voltage gated channel, Shaw-related subfamily, member 3 } \\
\text { potassium large conductance calcium-activated channel, subfamily M beta member } \\
\text { Kcnmb3 }\end{array}$ \\
XM_227040 & 0.23 & predicted) \\
Kv3.2; KShIIIA & NM_139217 & 0.32 & potassium voltage gated channel, Shaw-related subfamily, member 2 \\
Cnga3 & NM_053495 & 4.58 & cyclic nucleotide gated channel alpha 3 \\
Kcc2 & NM_134363 & 5.94 & solute carrier family 12, (potassium-chloride transporter) member 5 \\
Bec2 & NM_053630 & 6.16 & potassium voltage-gated channel, subfamily H (eag-related), member 4 \\
Hcn4 & NM_021658 & 9.79 & hyperpolarization-activated, cyclic nucleotide-gated K+ 4
\end{tabular}




\section{DISCUSSION}

We found that celecoxib and diclofenac induced wide-scale changes in the gene expression of neonatal rat cardiomyocytes. Pathway analysis of the microarray data showed changes in critical cell processes, including transcription, signal transduction and apoptosis. Genes encoding $\mathrm{Ca}^{+2}$ and $\mathrm{K}^{+}$channels were affected by both drugs. The different effects of these NSAIDs may be attributed to the fact that these drugs exhibit a wide range in IC50 inhibition of COX-1 and COX-2, due to differences in chemical structure and pharmacological properties. The duration of exposure to the drug was also an important factor.

NSAIDs were once commonly used in patients with degenerative osteoarthritis, rheumatoid arthritis, or ankylosing spondylitis. However, they require great attention because of side effects, such as gastro-intestinal ulceringor perforation and cardiovascular dysfunction. Some of these drugs have been under intense scrutiny since 2004, when Vioxx was voluntarily withdrawn from the market due to an increased risk of myocardial infarction and stroke in patients taking this medication for long periods (Marnett, 2009). These events are frequently associated with electrical instability and reduced contractile force of the ventricles (Yarishkin et al., 2009).

We previously reported that celecoxib, a selective COX-2 NSAID, interrupted excitation contraction coupling in cardiomyocytes (Baek et al., 2010). Another recent study shows that high doses of NSAIDs alters expression of genes regulating the cell cycle, proliferation, signal transduction, DNA damage and repair, apoptosis, autophagy and the inflammatory response (Palayoor et al., 2012). Recently, a study investigated the cardiovascular side effects of celecoxib and diclofenac and their effects on vascular smooth muscle through ion channels (Brueggemann et al., 2009). Several mechanisms are involved in cardiotoxicity including stress-response proteins (heat shock cognate $71 \mathrm{kDa}$ protein, mitochondrial stress 70 protein and mitochondrial heat shock protein 60), cytoskeletal proteins (myosin regulatory light chain 2 cardiac muscle isoform, tropomyosin $\alpha-1$ chain, myosin light chain 3, cardiac actin isoforms, prolyl 4-hyroxylase) and proteins involved in membrane assembly (Golgi reassembly-stacking protein 2 and septin-8) (Baek et al., 2010; Bläser et al., 2002; Takahashi et al., 2002). We found similar alterations in off-target gene expression, which may contribute to previously reported cardiotoxicity. These results are consistent with a previous study based on proteomic analysis of NSAIDs-treated primary cardiomyocytes (Baek et al., 2010).

Here, we found that expression of key genes related to $\mathrm{K}^{+}$channels $(\mathrm{Kcnmb} 1$ and $\mathrm{Kcnmb3}$ ) were down-regulated in celecoxib and diclofenac-treated rat cardiomyocytes, and other $\mathrm{Ca}^{+2}$ channels related genes were up-regulated or down-regulated. The combined effects of NSAIDs on L-type $\mathrm{Ca}^{+2}$ channels and $\mathrm{K}^{+}$channels may contribute to the cardiovascular side effects by vasodilation and/or vasoconstriction. It is uncertain whether the effects of celecoxib and diclofenac on $\mathrm{K}^{+}$channels and $\mathrm{Ca}^{+2}$ channels are dependent on and share a common signaling intermediate upstream pathway.

Generally, in vascular myocytes, suppression of $\mathrm{K}^{+}$currents typically leads to $\mathrm{Ca}^{+2}$ influx and vasoconstriction, whereas an increase in $\mathrm{K}^{+}$channel activity is typically associated with vasodilation (Standen and Quayle, 1998). As previously reported, Celecoxib inhibits voltage gated $\mathrm{K}^{+}$channels in several cell types, including rat retinal neurons and cardiac myocytes (Frolov et al., 2008). Brueggemann et al. (2009) also showed that 
celecoxib suppressed the larger $\mathrm{Kv}$ currents which become active at more positive potentials. However, they also reported that celecoxib induced dilation of arterial smooth muscle, suggesting that the enhancement of $\mathrm{K}^{+}$current and the suppression of L-type $\mathrm{Ca}^{+2}$ currents outweigh the inhibitory effect on $\mathrm{Kv}$ currents in cardiovascular smooth muscle. Inhibition of $\mathrm{K}^{+}$channels or activation of L-type voltage gated $\mathrm{Ca}^{+2}$ channels induces $\mathrm{Ca}^{+2}$ influx, smooth muscle contraction, and vasoconstriction (Standen and Quayle, 1998). In contrast, activation of $\mathrm{K}^{+}$channels or inhibition of L-type $\mathrm{Ca}^{+2}$ channels in cardiovascular smooth muscle accounts for the vasodilatory activity of celecoxib and diclofenac. Moreover, Sowers et al. (2005) reported that celecoxib reduces blood pressure by suppression of L-type $\mathrm{Ca}^{+2}$ channels, resulting in vasorelaxation (Sowers).

Our study focused mainly on genes related to cardiovascular function. Recently, array technology has been used for studying the side effects of NSAIDs on various organs (Tseng et al., 2002). In our study, we did not attempt to validate our results using in vitro experiments, which we recommend for further studies. Furthermore, our data show different gene expression patterns of the two drugs without giving any clear idea about possible mechanisms of action.

These experimental results could serve as the basis for further investigations to allow mechanistic insights into the biology of NSAIDs action. We suggest that this study could be used to help search for potential candidates for preclinical drug fingerprinting and to identify drug targets with a view towards optimizing drug efficacy and minimizing side effects.

In conclusion, this study provides a comprehensive analysis of gene expression in NSAIDs-treated cardiomyocytes. We provide clear evidence at the gene level that both types of ion signaling and MAPK signaling pathways are significantly affected by celecoxib and diclofenac. These findings provide a clue to explain, at least in part, how NSAIDs such as celecoxib and diclofenac cause side effects in the heart. These gene expression changes could be used to elect potential candidates for preclinical drug fingerprinting to identify drug targets with minimal side effects. Further studies are needed to demonstrate the role of these genes in human cardiomyocytes.

\section{ACKNOWLEDGEMENTS}

This research was supported by a grant from the Korean Food \& Drug Administration (08172KFDA507).

\section{CONFLICT OF INTEREST}

The authors report no conflicts of interest.

\section{REFERENCES}

Baek SM, Ahn JS, Noh HS, Park J, et al. (2010). Proteomic analysis in NSAIDs-treated primary cardiomyocytes. $J$. Proteomics 73: 721-732.

Barrett T and Edgar R (2006). Gene Expression Omnibus: Microarray Data Storage, Submission, Retrieval, and Analysis. Methods Enzymol. 411: 352-369.

Bläser S, Jersch K, Hainmann I, Wunderle D, et al. (2002). Human septin-septin interaction: CDCrel-1 partners with KIAA0202. FEBS Lett. 519: 169-172.

Brophy JM (2005). Cardiovascular Risk Associated with Celecoxib. N. Engl. J. Med. 352: 2648-2650. 
Brueggemann LI, Mackie AR, Mani BK, Cribbs LL, et al. (2009). Differential effects of selective cyclooxygenase-2 inhibitors on vascular smooth muscle ion channels may account for differences in cardiovascular risk profiles. Mol. Pharmacol. 76: 1053-1061.

Ferraz MB, Bombardier C, Day R, Hawkey CJ,et al. (2000). Comparison of upper gastrointestinal toxicity of rofecoxib and naproxen in patients with rheumatoid arthritis. VIGOR Study Group. N. Engl. J. Med. 343: 1520-1528.

Frolov RV, Berim IG and Singh S (2008a). Inhibition of delayed rectifier potassium channels and induction of arrhythmia: A novel effect of celecoxib and the mechanism underlying it. J. Biol. Chem. 283: 1518-1524.

Frolov RV, Slaughter MM and Singh S (2008b). Effects of celecoxib on ionic currents and spontaneous firing in rat retinal neurons. Neuroscience 154: 1525-1532.

Jones MK, Wang H, Peskar BM, Levin E, et al. (1999). Inhibition of angiogenesis by nonsteroidal anti-inflammatory drugs: insight into mechanisms and implications for cancer growth and ulcer healing. Nat. Med. 5: 1418-1423.

Lin H-P, Kulp SK, Tseng P-H, Yang Y-T, et al. (2004). Growth inhibitory effects of celecoxib in human umbilical vein endothelial cells are mediated through G1 arrest via multiple signaling mechanisms. Mol. Cancer Ther. 3: 16711680.

Mackie AR and Byron KL (2008). Cardiovascular KCNQ (Kv7) potassium channels: physiological regulators and new targets for therapeutic intervention. Mol. Pharmacol. 74: 1171-1179.

Marnett LJ (2009). The COXIB experience: a look in the rearview mirror. Annu. Rev. Pharmacol. Toxicol. 49: 265-290.

Palayoor ST, J-Aryankalayil M, Makinde AY, Cerna D, et al.(2012). Gene expression profile of coronary artery cells treated with nonsteroidal anti-inflammatory drugs reveals off-target effects. J. Cardiovasc. Pharmacol. 59: 487499.

Raju U, Nakata E, Yang P, Newman RA,et al. (2002). In vitro enhancement of tumor cell radiosensitivity by a selective inhibitor of Cyclooxygenase-2 enzyme: mechanistic considerations. Int. J. Radiat. Oncol. 54: 886-894.

Rodgers LS, Schnurr DC, Broka D and Camenisch TD (2009). An improved protocol for the isolation and cultivation of embryonic mouse myocytes. Cytotechnology 59: 93-102.

Schjerning Olsen AM, Fosbøl EL, Lindhardsen J, Folke F, et al. (2011). Duration of treatment with non-steroidal antiinflammatory drugs and impact on risk of death and recurrent myocardial infarction in patients with prior myocardial infarction: a nationwide cohort study. Circulation 123: 2226-2235.

Smyth GK, Speed T (2003). Normalization of cDNA microarray data. Methods 31: 265-273.

Sowers JR, White WB, Pitt B, Whelton A,et al. (2005). The effects of cyclooxygenase-2 inhibitors and nonsteroidal antiinflammatory therapy on 24-hour blood pressure in patients with Hypertension, Osteoarthritis, and Type 2 Diabetes Mellitus. Arch. Intern. Med. 165: 161.

Standen NB and Quayle JM (1998). K ${ }^{+}$channel modulation in arterial smooth muscle. Acta Physiol. Scand. 164: 549557.

Takahashi S, Dohi N, Takahashi Y and Miura T (2002). Cloning and characterization of the 5'-flanking region of the rat P4H $\alpha$ gene encoding the prolyl 4-hydroxylase $\alpha(\mathrm{I})$ subunit. Biochim. Biophys. Acta - Gene Struct. Expr. 1574: 354358.

Tegeder I, Pfeilschifter J and Geisslinger G (2001). Cyclooxygenase-independent actions of cyclooxygenase inhibitors. FASEB J. 15: 2057-2072.

Tseng WW, Deganutti A, Chen MN, Saxton RE, et al. (2002). Selective cyclooxygenase-2 inhibitor rofecoxib (Vioxx) induces expression of cell cycle arrest genes and slows tumor growth in human pancreatic cancer. J. Gastrointest. Surg. 6: 838-843.

Wei D, Wang L, He Y, Xiong HQ, et al. (2004). Celecoxib inhibits vascular endothelial growth factor expression in and reduces angiogenesis and metastasis of human pancreatic cancer via suppression of Sp1 transcription factor activity. Cancer Res. 64: 2030-2038.

Wolfe MM, Lichtenstein DR and Singh G (1999). Gastrointestinal toxicity of non-steroidal anti-inflammatory drugs. $N$. Engl. J. Med. 340: 1888-1899.

Yarishkin OV, Hwang EM, Kim D, Yoo JC, et al. (2009). Diclofenac, a non-steroidal anti-inflammatory drug, inhibits Ltype Ca channels in neonatal rat ventricular cardiomyocytes. Korean J. Physiol. Pharmacol. 13: 437-442.

Yin T, Wang G, Ye T and Wang Y (2016). Sulindac, a non-steroidal anti-inflammatory drug, mediates breast cancer inhibition as an immune modulator. Sci. Rep. 6: 19534. 